\title{
ON SOME SEMILINEAR EQUATIONS OF SCHRÖDINGER TYPE
}

\author{
ROSSELLA AGLIARDI and DANIELA MARI
}

\begin{abstract}
We study the initial value problem for some semilinear pseudo-differential equations of the form $\partial_{t} u+i H\left(x, D_{x}\right) u=F(u, \nabla u)$. The assumptions we make on $H$ are trivially satisfied by $\Delta$, thus our equations generalize Schrödinger type equations. A local existence theorem is proved in some weighted Sobolev spaces.
\end{abstract}

\section{Introduction}

In this paper we consider the initial value problem for some nonlinear evolution equations of the form

$$
\partial_{t} u+i H\left(x, D_{x}\right) u=F(u, \nabla u)
$$

where $H$ is a uniformly elliptic pseudo-differential operator of order 2 with real symbol.

We assume that the nonlinear term $F: \mathrm{C} \times \mathrm{C}^{n} \rightarrow \mathrm{C}$ satisfies: $F(u, q) \in$ $\mathscr{C}^{\infty}\left(\mathrm{R}^{2} \times \mathrm{R}^{2 n}\right)$ and $|F(u, q)| \leq C\left(|u|^{2}+|q|^{2}\right)$ near the origin.

The simplest model we have in mind is the one with $H(x, \xi)=|\xi|^{2}$, that is (1) generalizes semilinear Schrödinger equations.

Most papers on semilinear Schrödinger equations are concerned with the case $F(u)$ or $F(u, \nabla u)$ but $\operatorname{Im} \frac{\partial F}{\partial q_{j}}=0, j=1, \ldots, n$. Some troubles arise when one works with classical energy methods in the general case: even in the linear case some difficulties arise owing to the imaginary part of the coefficients of $\partial_{x_{j}} u$. Correspondingly all the papers about the wellposedness of the Cauchy problem in $L^{2}$ or Sobolev spaces for linear Schrödinger equations give necessary or sufficient conditions on the imaginary part of the first order terms of the operator. (See [7], [8], [9], [12]).

In [2] Chihara succeeded in proving local existence in some weighted Sobolev spaces for the semilinear Schrödinger equations in the case $n=1$. In [3] he generalized the result to higher space dimension. Our paper studies more

Received June 16, 1998; in revised form February 11, 1999. 
general operators of Schrödinger type and thus it generalizes [3]. We need the following additional assumption on $H$ :

$$
\exists c>0 \text { such that }\{H, p\}(x, \xi) \geq c\langle x\rangle^{-2}|\xi| \text { for large }|\xi|,
$$

where $p(x, \xi)=\langle\xi\rangle^{-1} \sum_{j=1}^{n} \xi_{j} \operatorname{arctg} x_{j}$ and $\{.,$.$\} denotes the Poisson's brac-$ ket, i.e. $\{H, p\}=\sum_{j=1}^{n}\left(\partial_{\xi_{j}} H \partial_{x_{j}} p-\partial_{\xi_{j}} p \partial_{x_{j}} H\right)$.

A condition similar to (2) can be found in the literature on Schrödinger equations (see (A2) in [5] for example). Such conditions are used to eliminate - in some sense - the bad first order term.

\section{Notation}

For $x \in \mathrm{R}^{n}$ let $\langle x\rangle=\left(1+|x|^{2}\right)^{1 / 2}$ and $\left\langle D_{x}\right\rangle=\left(1-\Delta_{x}\right)^{1 / 2}$.

Let \|\| denote the $L^{2}$-norm.

For $m, p \in \mathrm{R}$ let $\|f\|_{m, p}=\left\|\langle x\rangle^{p}\left\langle D_{x}\right\rangle^{m} f\right\|$ and let $H^{m, p}=\left\{f \in \mathscr{S}^{\prime}\left(\mathrm{R}^{n}\right)\right.$; $\left.\|f\|_{m, p}<\infty\right\}$.

Note that $H^{m, 0}$ is the usual Sobolev space $H^{m}$.

In the sequel if $\ell$ is a sufficiently large integer we shall denote $H^{m+\ell, 0} \cap$ $H^{m+1,1} \cap H^{m, 2}$ by $\Xi^{m, \ell}$.

We shall use the following notation for pseudo-differential operators. The space of the symbols $\sigma(x, \xi) \in \mathscr{C}^{\infty}\left(\mathrm{R}^{n} \times \mathrm{R}^{n}\right)$ such that

$$
\sup _{\substack{x, \xi \in \in^{n} \\ \alpha, \beta \in \mathbb{N}^{n}}}\left|\partial_{\xi}^{\alpha} D_{x}^{\beta} \sigma(x, \xi)\right|\langle\xi\rangle^{|\alpha|-m}<\infty
$$

will be denoted by $S^{m}$. The calculus for the corresponding pseudo-differential operators can be found in Kumano-go's book [11].

\section{The main result}

Consider the following Cauchy problem for an equation of Schrödinger type:

$$
\left.\left.\partial_{t} u+i H\left(x, D_{x}\right) u=F\left(u, \nabla_{x} u\right) \quad \text { in } \quad\right] 0, \infty\right) \times \mathrm{R}^{n}, u(t=0)=u_{\circ}
$$

We make the following assumptions:

(H1) $H$ has a real symbol;

(H2) there exists $c_{\circ}>0$ such that $|H(x, \xi)| \geq c_{\circ}|\xi|^{2} \forall x, \xi \in \mathrm{R}^{n}$;

(H3) $\exists c>0$ such that $\{H, p\}(x, \xi) \geq c\langle x\rangle^{-2}|\xi|$ for large $|\xi|$, where $\{.,$. denotes the Poisson's bracket and $p(x, \xi)=\langle\xi\rangle^{-1} \sum_{j=1}^{n} \xi_{j} \operatorname{arctg} x_{j}$.

(H4) $\sup _{x, \xi \in \mathrm{R}^{n}}\left|\partial_{\xi}^{\alpha} D_{x}^{\beta} H(x, \xi)\right| .\langle\xi\rangle^{|\alpha+\beta|-2}<\infty, \quad \forall \alpha, \beta \in \mathrm{N}^{n}$. 
Moreover we make the following assumptions on the nonlinear term:

(F1) $F: \mathrm{C} \times \mathrm{C}^{n} \rightarrow \mathrm{C}$ belongs to $\mathscr{C}^{\infty}\left(\mathrm{R}^{2} \times \mathrm{R}^{2 n}\right)$;

(F2) there exists $C>0$ such that $|F(u, q)| \leq C\left(|u|^{2}+|q|^{2}\right)$ near $(u, q)=$ $(0,0)$.

In the following section we prove the following

Theorem 2.1. For any initial datum $u_{\circ} \in \Xi^{m, \ell}$ (where $m$ and $\ell$ are sufficiently large integers) there exists a time $T>0$ such that the Cauchy problem (3) has a solution $u \in \mathscr{C}\left([0, T] ; \Xi^{m, \ell}\right)$.

To prove this theorem at first we consider a parabolic regularization of our problem which depends on a viscosity parameter $\varepsilon>0$. The regularized problem is solved by linearization in §4. Finally a solution of (3) is obtained as a zero limit of the solution of the regularized problem.

\section{Parabolic regularization}

For any $\varepsilon \in] 0,1]$ let us consider

$$
\left\{\begin{array}{l}
\partial_{t} u^{\varepsilon}-\varepsilon \Delta_{x} u^{\varepsilon}+i H\left(x, D_{x}\right) u^{\varepsilon}=F\left(u^{\varepsilon}, \nabla_{x} u^{\varepsilon}\right) \\
u^{\varepsilon}(0, x)=u_{o}(x)
\end{array}\right.
$$

in $] 0,+\infty) \times \mathrm{R}^{n}$, where $H, F$ and $u_{\circ}$ are as in $\S 2$.

Let $P_{\varepsilon}$ denote the linear operator $\partial_{t}-\varepsilon \Delta_{x}+i H\left(x, D_{x}\right)$. Let us first construct a fundamental solution $S_{\varepsilon}(t)$ for $P_{\varepsilon}$. Consider the following eikonal equation:

$$
\left\{\begin{array}{l}
\partial_{t} \phi(t, s ; x, \xi)+H\left(x, \nabla_{x} \phi(t, s ; s, \xi)\right) \\
\phi(s, s ; x, \xi)=x . \xi
\end{array}\right.
$$

Then we have the following

Lemma 3.1. If $H$ satisfies (H1) and (H4), then there exists $T>0$ such that for every $t, s \in[-T, T]$ the following estimate is true:

$$
\sup _{x \in \mathrm{R}^{n}}\left|\partial_{\xi}^{\alpha} \partial_{x}^{\beta}(\phi(t, s ; x, \xi)-x . \xi)\right| \leq C_{\alpha, \beta}^{\prime}|t-s|\langle\xi\rangle^{2-|\alpha+\beta|}
$$

$\forall \alpha, \beta \in \mathrm{N}^{n}, \forall \xi \in \mathrm{R}^{n}$ with large $|\xi|$, and for some $C_{\alpha, \beta}^{\prime}$.

Proof. The proof follows the lines of Theorem 4.1 in [11]. At first we prove inductively that the solutions $q(t, s ; y, \xi)$ and $p(t, s ; y, \xi)$ of the Hamilton's equations

$$
\left\{\begin{array}{l}
\frac{d q}{d t}=\nabla_{\xi} H(q, p) \quad \frac{d p}{d t}=-\nabla_{x} H(q, p) \\
\left.(q, p)\right|_{t=s}=(y, \xi)
\end{array}\right.
$$


satisfy the following estimates, for every $\alpha, \beta \in \mathrm{N}^{n}$ :

$$
\begin{aligned}
& \sup _{y \in \mathrm{R}^{n}}\left|\partial_{\xi}^{\alpha} \partial_{y}^{\beta}(q(t, s ; y, \xi)-y)\right| \leq C_{\alpha, \beta}^{\prime \prime}|t-s|\langle\xi\rangle^{1-|\alpha+\beta|} \\
& \sup _{y \in \mathrm{R}^{n}}\left|\partial_{\xi}^{\alpha} \partial_{y}^{\beta}(p(t, s ; x, \xi)-\xi)\right| \leq C_{\alpha, \beta}^{\prime \prime}|t-s|\langle\xi\rangle^{1-|\alpha+\beta|}
\end{aligned}
$$

Denoting the inverse mapping of $y \rightarrow x=q(t, s ; y, \xi)$ by $Y(t, s ; x, \xi)$, we can prove that, if $T>0$ is sufficiently small, then for every $\alpha, \beta \in \mathrm{N}^{n}$, $t, s \in[-T, T], \xi \in \mathrm{R}^{n}$ with large $|\xi|$, and for some $A_{\alpha, \beta}$, the following inequality holds:

$$
\sup _{y \in R^{n}}\left|\partial_{\xi}^{\alpha} \partial_{x}^{\beta}(Y(t, s ; x, \xi)-x)\right| \leq A_{\alpha, \beta}|t-s|\langle\xi\rangle^{1-|\alpha+\beta|}
$$

Finally we construct the solution of (5) setting

$$
\phi(t, s ; x, \xi)=\psi(t, s ; Y(t, s ; x, \xi), \xi),
$$

where

$$
\psi(t, s ; y, \xi)=y . \xi+\int_{s}^{t}\left(p . \nabla_{\xi} H-H\right)(\tau, q(\tau, s ; y, \xi), p(\tau, s ; y, \xi)) d \tau .
$$

Consequently, we get (6).

Now we are going to construct a Fourier integral operator whose phase is $\phi(t, s ; x, \xi)$ and whose amplitude $\sigma(t, s ; x, \xi) \sim \sum_{j=0}^{\infty} \sigma_{2 j}(t, s ; x, \xi)$ is found by solving the following transport equations:

$\left(T_{0}\right)\left\{\begin{array}{l}\partial_{t} \sigma_{0}(t)+\nabla_{\xi} H\left(x, \nabla_{x} \phi(t, s ; x, \xi)\right) \cdot \nabla_{x} \sigma_{0}(t)+c_{\varepsilon}(t, x, \xi) \sigma_{0}(t)=0 \\ \sigma_{0}(s)=1\end{array}\right.$

where

$$
\begin{aligned}
& c_{\varepsilon}(t, x, \xi) \\
& \quad=\frac{1}{2} \sum_{k i} \partial_{\xi_{k} \xi_{i}}^{2} H\left(x, \nabla_{x} \phi(t, s ; x, \xi)\right) \partial_{x_{k} x_{i}}^{2} \phi(t, s ; x, \xi)+\varepsilon\left|\nabla_{x} \phi(t, s ; x, \xi)\right|^{2},
\end{aligned}
$$

and for $j \geq 1$

$$
\left(T_{2 j}\right)\left\{\begin{aligned}
\partial_{t} \sigma_{2 j}(t)+\nabla_{\xi} H\left(x, \nabla_{x} \phi(t, s ; x, \xi)\right) . \nabla_{x} \sigma_{2 j}(t) & \\
& +c_{\varepsilon}(t, x, \xi) \sigma_{2 j}(t)=-i b_{j}(t, x, \xi) \\
\sigma_{2 j}(s)=0 &
\end{aligned}\right.
$$


with

$$
\begin{aligned}
& b_{j}(t, x, \xi) \\
& =\sum_{k=1}^{j} \sum_{|\gamma|=k+1} \frac{1}{\gamma !} D_{z}^{\gamma}\left\{\partial_{\xi}^{\gamma} H\left(x, \tilde{\nabla}_{x} \phi(t, s ; x, z, \xi)\right) \sigma_{2 j-2 k}(t, s ; z, \xi)\right\}_{z=x} \\
& \quad-2 \varepsilon \nabla_{x} \phi(t, s ; x, \xi) . \nabla_{x} \sigma_{2 j-2}(t, s ; x, \xi) \\
& \quad+i \varepsilon \Delta_{x} \sigma_{2 j-2}(t, s ; x, \xi) \\
& \quad-\varepsilon \Delta_{x} \phi(t, s ; x, \xi) \sigma_{2 j-2}(t, s ; x, \xi)
\end{aligned}
$$

being $\tilde{\nabla}_{x} \phi(t, s ; x, z, \xi)=\int_{0}^{1} \nabla_{x} \phi(t, s ; \theta z+(1-\theta) x, \xi) d \theta$.

We can prove inductively that there exists an increasing sequence $C_{n}^{*}$ such that:

$$
\begin{array}{r}
\left|\partial_{\xi}^{\alpha} \partial_{x}^{\beta} \sigma_{2 j}(t, s ; x, \xi)\right| \leq \exp \left(-3 \varepsilon|t-s||\xi|^{2} / 4\right) \cdot C_{*}^{|\alpha+\beta|+6 j}\langle\xi\rangle^{-|\alpha+\beta|-2 j} \\
\cdot \sum_{k=0}^{|\alpha+\beta|+2 j} \frac{\left\{2 \varepsilon|t-s||\xi|^{2}\right\}^{k}}{k !}
\end{array}
$$

for every $\alpha, \beta \in \mathrm{N}^{n}$ and for every $j \in \mathrm{N}$. We can write:

$$
\sum_{k=0}^{|\alpha+\beta|+2 j} \frac{\left\{2 \varepsilon|t-s||\xi|^{2}\right\}^{k}}{k !} \leq 8^{|\alpha+\beta|+2 j} \exp \left(\varepsilon|t-s||\xi|^{2} / 4\right),
$$

so that (7) becomes:

$$
\left|\partial_{\xi}^{\alpha} \partial_{x}^{\beta} \sigma_{2 j}(t, s ; x, \xi)\right| \leq \exp \left(-\varepsilon|t-s||\xi|^{2} / 2\right) C_{\alpha, \beta, j}^{* *}\langle\xi\rangle^{-|\alpha+\beta|-2 j} .
$$

Finally, as in Lemma 3.2 in [11], we can construct a symbol which is equivalent to the formal series of the symbols $\sigma_{2 j}$. Thus we obtain a fundamental solution of $P_{\varepsilon}$ in the form of a Fourier integral operator $S^{\varepsilon}(t)$ with phase $\phi$ and amplitude $\sigma^{\varepsilon}$ such that:

$$
\left|\partial_{\xi}^{\alpha} \partial_{x}^{\beta} \sigma^{\varepsilon}(t, s ; x, \xi)\right| \leq \exp \left(-\varepsilon|t-s||\xi|^{2} / 2\right) \cdot C_{\alpha, \beta}\langle\xi\rangle^{-|\alpha+\beta|} .
$$

Now we can prove the following

PROPOSITION 3.2. If $m, \ell$ are sufficiently large then for any $u_{o} \in \Xi^{m, \ell}$ there exists a time $T_{\varepsilon}=T\left(\varepsilon,\left\|u_{o}\right\|_{\Xi} m, \ell\right)>0$ such that (4) has a unique solution $u^{\varepsilon} \in \mathscr{C}\left(\left[0, T_{\varepsilon}\right] ; \Xi^{m, \ell}\right)$. 
Proof. Let $\varphi(x)$ be $1, x_{j}(j=1, \ldots, n)$ or $|x|^{2}$ and let $\alpha \in \mathrm{N}^{n}$ be such that

$$
|\alpha| \leq \begin{cases}m+\ell & \text { if } \phi(x)=1 \\ m+1 & \text { if } \phi(x)=x_{j} \\ m & \text { if } \phi(x)=|x|^{2}\end{cases}
$$

We fix $u$ in a class that will be defined in the continuation of this proof and consider

$$
\left\{\begin{array}{l}
\partial_{t} v-\varepsilon \Delta_{x} v+i H\left(x, D_{x}\right) v=F\left(u, \nabla_{x} u\right) \\
v(0, x)=u_{o}(x)
\end{array}\right.
$$

Applying $\varphi(x) \partial_{x}^{\alpha}$ to (9) we get:

$$
\begin{array}{r}
\partial_{t}\left(\varphi(x) \partial_{x}^{\alpha} v\right)-\varepsilon \Delta_{x}\left(\varphi(x) \partial_{x}^{\alpha} v\right)+i H\left(x, D_{x}\right)\left(\varphi(x) \partial_{x}^{\alpha} v\right) \\
=-\varepsilon\left(\Delta_{x} \varphi(x) \partial_{x}^{\alpha} v+2 \nabla_{x} \varphi(x) . \nabla_{x} \partial_{x}^{\alpha} v\right)-i\left[\varphi(x) \partial_{x}^{\alpha}, H\left(x, D_{x}\right)\right] v \\
+\varphi(x) \partial_{x}^{\alpha} F\left(u, \nabla_{x} u\right)
\end{array}
$$

and

$$
\varphi(x) \partial_{x}^{\alpha} v(0, x)=\varphi(x) \partial_{x}^{\alpha} u_{o}(x),
$$

where $[.,$.$] denotes the usual commutator.$

Let us consider the fundamental solution $S^{\varepsilon}(t)$ of $P_{\varepsilon}$ that we constructed above. Then going back to (10) we can write:

$$
\begin{aligned}
\varphi \partial_{x}^{\alpha} v(t)=S^{\varepsilon}(t)\left(\varphi \partial_{x}^{\alpha} u_{o}\right)+\varepsilon \int_{0}^{t} S^{\varepsilon}(t-\tau)\left(\Delta_{x} \varphi \partial_{x}^{\alpha} v+2 \nabla_{x} \varphi \cdot \nabla_{x} \partial_{x}^{\alpha} v\right)(\tau) d \tau \\
-i \int_{0}^{t} S^{\varepsilon}(t-\tau)\left[\varphi \partial_{x}^{\alpha}, H\left(x, D_{x}\right)\right] v(\tau) d \tau \\
+\int_{0}^{t} S^{\varepsilon}(t-\tau)\left(\varphi \partial_{x}^{\alpha} F\left(u, \nabla_{x} u\right)\right)(\tau) d \tau
\end{aligned}
$$

Let $\Phi^{\varepsilon}$ be a solution operator of (9) defined by $\Phi^{\varepsilon}(u)=v$; then

$$
\begin{aligned}
\varphi \partial_{x}^{\alpha} \Phi^{\varepsilon}(u)(t)= & S^{\varepsilon}(t)\left(\varphi \partial_{x}^{\alpha} u_{o}\right) \\
& +\varepsilon \int_{0}^{t} S^{\varepsilon}(t-\tau)\left(\Delta_{x} \varphi \partial_{x}^{\alpha} \Phi^{\varepsilon}(u)+2 \nabla_{x} \varphi \cdot \nabla_{x} \partial_{x}^{\alpha} \Phi^{\varepsilon}(u)\right)(\tau) d \tau \\
& -i \int_{0}^{t} S^{\varepsilon}(t-\tau)\left[\varphi \partial_{x}^{\alpha}, H\left(x, D_{x}\right)\right] \Phi^{\varepsilon}(u)(\tau) d \tau \\
& +\int_{0}^{t} S^{\varepsilon}(t-\tau)\left(\varphi \partial_{x}^{\alpha} F\left(u, \nabla_{x} u\right)\right)(\tau) d \tau
\end{aligned}
$$


Taking (8) into account and adapting Th. 2.3 in Ch. 10 of [11] we obtain, for some constant $c_{\sigma}>0$, the following estimate:

$$
\left\|\varphi \partial_{x}^{\alpha} \Phi^{\varepsilon}(u)(t)\right\| \leq c_{\sigma}\left(\left\|\varphi \partial_{x}^{\alpha} u_{o}\right\|+\varepsilon \int_{0}^{t} I_{1}(\tau) d \tau+\int_{0}^{t} I_{H}(\tau) d \tau\right)+\int_{0}^{t} I_{F}(\tau) d \tau,
$$

where

$$
\begin{aligned}
I_{1}(\tau) & =\left\|\Delta_{x} \varphi \partial_{x}^{\alpha} \Phi^{\varepsilon}(u)(\tau)\right\|+2\left\|\nabla_{x} \varphi \cdot \nabla_{x} \partial_{x}^{\alpha} \Phi^{\varepsilon}(u)(\tau)\right\| \\
I_{H}(\tau) & =\left\|\left[\varphi \partial_{x}^{\alpha}, H\left(x, D_{x}\right)\right] \Phi^{\varepsilon}(u)(\tau)\right\| \\
I_{F}(\tau) & =\left\|S^{\varepsilon}(t-\tau)\left(\varphi(x) \partial_{x}^{\alpha} F\left(u, \nabla_{x} u\right)\right)(\tau)\right\| .
\end{aligned}
$$

Let $B_{r}(T)=\left\{u \in L^{\infty}([0, T]), \Xi^{m, \ell}\right) ;\|u\|_{m, \ell, T}=\sup _{t \in[0, T]}\|u(t)\|_{\Xi} m, \ell \leq$ $r$ w where $r>0$ is such that $\left\|u_{o}\right\|_{\Xi} m, \ell<r /\left(2 c_{\sigma}\right)$, and assume $u \in B_{r}(T)$. It follows immediately that

$$
I_{1}(\tau) \leq c^{\prime}\left\|\Phi^{\varepsilon}(u)\right\|_{m, \ell, T}
$$

and since, in view of (H4), we can write

$$
\left[\varphi(x) \partial_{x}^{\alpha}, H\left(x, D_{x}\right)\right]=\varphi(x) R_{|\alpha|}\left(x, D_{x}\right)+\nabla \varphi(x) \cdot R_{|\alpha|+1}^{\prime}\left(x, D_{x}\right)+R_{\alpha \mid}^{\prime \prime}\left(x, D_{x}\right),
$$

where the subscripts denote the order of the operators, then we have

$$
I_{H}(\tau) \leq C^{\prime \prime}\left\|\Phi^{\varepsilon}(u)\right\|_{m, \ell, T} .
$$

If we choose $\varphi(x)=1, x_{j},|x|^{2}$ and $|\alpha|<m+\ell, m+1, m$ respectively, then we have:

$$
I_{F}(\tau) \leq C_{r}^{\prime}\left\|\varphi\left\langle D_{x}\right\rangle^{|\alpha|+1} u(\tau)\right\| \leq C_{r}^{\prime \prime}\|u(\tau)\|_{\Xi} m, \ell .
$$

In the cases $|\alpha|=m+\ell, m+1, m$ respectively, we can obtain the following estimates. Let $\hat{\alpha}=\left(\alpha_{1}, \ldots, \alpha_{k}-1, \ldots, \alpha_{n}\right)$ for some $k \in\{1, \ldots, n\}$. Then

$$
\begin{aligned}
& I_{F}(\tau) \leq\left\|S^{\varepsilon}(t-\tau)\left(\partial_{x_{k}}\left(\varphi \partial_{x}^{\hat{\alpha}} F\left(u, \nabla_{x} u\right)\right)(\tau)\right)\right\| \\
& \quad+\left\|S^{\varepsilon}(t-\tau)\left(\partial_{x_{k}} \varphi \partial_{x}^{\hat{\alpha}} F\left(u, \nabla_{x} u\right)(\tau)\right)\right\| \\
& \leq \sup _{\xi \in \mathrm{R}^{n}}\left(|\xi| e^{-\varepsilon|\xi|^{2}(t-\tau) / 2}\right) \hat{C}\left\|\varphi \partial_{x}^{\hat{\alpha}} F\left(u, \nabla_{x} u\right)(\tau)\right\| \\
& \quad+c_{\sigma}\left\|\partial_{x_{k}} \varphi \partial_{x}^{\hat{\alpha}} F\left(u, \nabla_{x} u\right)(\tau)\right\| \\
& \leq \hat{C} /(\sqrt{\varepsilon(t-\tau)})\left\|\varphi \partial_{x}^{\hat{\alpha}} F\left(u, \nabla_{x} u\right)(\tau)\right\|+c_{\sigma}\left\|\partial_{x_{k}} \varphi \partial_{x}^{\hat{\alpha}} F\left(u, \nabla_{x} u\right)(\tau)\right\| \\
& \leq \tilde{C}_{r}(1+1 / \sqrt{\varepsilon(t-\tau)})\|u(\tau)\|_{\Xi} m, \ell .
\end{aligned}
$$


Summing up we get the following estimate:

$$
\left\|\Phi^{\varepsilon}(u)\right\|_{m, \ell, T} \leq c_{\sigma}\left\|u_{o}\right\|_{\Xi} m, \ell+C^{*} T\left\|\Phi^{\varepsilon}(u)\right\|_{m, \ell, T}+C_{r}(T+2 \sqrt{T / \varepsilon}) r .
$$

Hence, if we choose a sufficiently small $T_{\varepsilon}$, we get

$$
\left\|\Phi^{\varepsilon}(u)\right\|_{m, \ell, T} \leq r \quad \forall T \leq T_{\varepsilon} .
$$

If $u, u^{\prime} \in B_{r}(T)$ a similar computation gives:

$$
\left\|\Phi^{\varepsilon}(u)-\Phi^{\varepsilon}\left(u^{\prime}\right)\right\|_{m, \ell, T} \leq\left(C_{r} /\left(1-C^{*} T\right)\right)(T+\sqrt{T / \varepsilon})\left\|u-u^{\prime}\right\|_{m, \ell, T} .
$$

Then $\Phi^{\varepsilon}$ is a contraction mapping on $B_{r}(T), \forall T \leq T_{\varepsilon}$.

\section{Linearization and uniform energy estimates}

In this section we write (4) in the form of a system. Then we diagonalize the system. Finally we are able to obtain energy estimates by applying a method which is now almost classic in the theory of linear equations of Schrödinger type.

Let $w={ }^{t}\left(\varphi \partial_{x}^{\alpha} u, \varphi \partial_{x}^{\alpha} \bar{u}\right)$. Then (4) can be written in the following form:

$$
\left(\partial_{t}-\varepsilon \Delta+i \mathscr{H}-i \mathscr{B}\right) w=G(u)
$$

where

$$
\begin{aligned}
& \mathscr{H}\left(x, D_{x}\right)=\left(\begin{array}{cc}
H\left(x, D_{x}\right) & 0 \\
0 & -H\left(x, D_{x}\right)
\end{array}\right) \\
& \mathscr{B}\left(x, D_{x}\right)=\left(\begin{array}{cc}
\sum_{j=1}^{n} \frac{\partial F}{\partial q_{j}}(u, \nabla u) D_{x_{j}} & \sum_{j=1}^{n} \frac{\partial F}{\partial \bar{q}_{j}}(u, \nabla u) D_{x_{j}} \\
\sum_{j=1}^{n} \frac{\partial F}{\partial q_{j}}(u, \nabla u) D_{x_{j}} & \sum_{j=1}^{n} \frac{\partial F}{\partial \bar{q}_{j}}(u, \nabla u) D_{x_{j}}
\end{array}\right)
\end{aligned}
$$


and $G(u)={ }^{t}(g(u), \overline{g(u)})$ with

$g(u)$

$$
\begin{aligned}
=- & \varepsilon\left(\Delta_{x} \varphi(x) \partial_{x}^{\alpha} u+2 \nabla_{x} \varphi(x) . \nabla_{x} \partial_{x}^{\alpha} u\right)-i\left[\varphi(x) \partial_{x}^{\alpha}, H\left(x, D_{x}\right)\right] u \\
+ & \varphi(x) \sum_{\gamma \leq \hat{\alpha}}\left(\begin{array}{c}
\hat{\alpha} \\
\gamma
\end{array}\right)\left(\partial_{x}^{\gamma}\left(\frac{\partial F}{\partial u}\left(u, \nabla_{x} u\right)\right) \partial_{x}^{\alpha-\gamma}+\partial_{x}^{\gamma}\left(\frac{\partial F}{\partial \bar{u}}\left(u, \nabla_{x} u\right)\right) \partial_{x}^{\alpha-\gamma} \bar{u}\right) \\
+ & \varphi(x) \sum_{j=1}^{n} \sum_{0<\gamma \leq \hat{\alpha}}\left(\begin{array}{l}
\hat{\alpha} \\
\gamma
\end{array}\right)\left(\partial_{x}^{\gamma}\left(\frac{\partial F}{\partial q_{j}}\left(u, \nabla_{x} u\right)\right) \partial_{x_{j}} \partial^{\alpha-\gamma} u\right. \\
& \left.+\partial_{x}^{\gamma}\left(\frac{\partial F}{\partial \bar{q}_{j}}\left(u, \nabla_{x} u\right)\right) \partial_{x_{j}} \partial^{\alpha-\gamma} \bar{u}\right) \\
& -\sum_{j=1}^{n} \partial_{x_{j}} \varphi(x)\left(\frac{\partial F}{\partial q_{j}}\left(u, \nabla_{x} u\right) \partial_{x}^{\alpha} u+\frac{\partial F}{\partial \bar{q}_{j}}\left(u, \nabla_{x} u\right) \partial_{x}^{\alpha} \bar{u}\right)
\end{aligned}
$$

if $|\alpha|>0$ and $\hat{\alpha}=\left(\alpha_{1}, \ldots, \alpha_{k}-1, \ldots\right)$ for some $k \in\{1, \ldots, n\}$.

Let $u(t) \in \Xi^{m, \ell}$ be such that $\sup _{t \in[0, T]}\|u(t)\|_{\Xi^{m-1, \ell}} \leq r$. Since $F$ is quadratic, there exists a constant $c_{r}$ such that

$$
\begin{aligned}
\left|\frac{\partial F}{\partial q_{j}}(u, \nabla u)(t, x)\right| & \leq c_{r}\left(|u(t, x)|+\left|\nabla_{x} u(t, x)\right|\right) \\
& \leq C c_{r}\langle x\rangle^{-2}\left\|\langle x\rangle^{2} u(t, x)\right\|_{H^{[n / 2]+2}} \\
& \leq C c_{r}\langle x\rangle^{-2}\|u(t)\|_{\Xi^{m-1, \ell}}
\end{aligned}
$$

if $m \geq[n / 2]+3$ and analogously

$$
\left|\frac{\partial F}{\partial \bar{q}_{j}}(u, \nabla u)(t, x)\right| \leq C c_{r}\langle x\rangle^{-2}\|u(t)\|_{\Xi^{m-1, \ell}} .
$$

Moreover taking (14) into account we can prove

$$
\|G(u(t))\| \leq C_{r}^{\prime}\|u(t)\|_{\Xi^{m, \ell}} .
$$

Now define the operator $L(t)=L\left(t, x, D_{x}\right)$ whose symbol is

$$
\ell(t, x, \xi)=\left(\begin{array}{cc}
H(x, \xi)-b_{11}(t, x, \xi) & -b_{12}(t, x, \xi) \\
-b_{21}(t, x, \xi) & -H(x, \xi)-b_{22}(t, x, \xi)
\end{array}\right)
$$


where $\left(b_{i k}\right)_{i, k=1,2}$ are the entries of $\mathscr{B}$. Note that $b_{i k}(t, x, \xi)=\sum_{j=1}^{n} b_{i k j}(t, x) \xi_{j}$ with $\left|b_{i k j}(t, x)\right| \leq r c_{r}\langle x\rangle^{-2} \forall t \in[0, T]$ in view of (14). Let

$$
\tilde{\lambda}(t, x, \xi)=\left(\begin{array}{cc}
0 & \frac{1}{2} b_{12}(t, x, \xi) / H(x, \xi) \\
-\frac{1}{2} b_{21}(t, x, \xi) / H(x, \xi) & 0
\end{array}\right)
$$

In view of $(\mathrm{H} 2) \tilde{\lambda}(t) \in\left(S^{-1}\right)^{2 \times 2} \forall t \in[0, T]$. Let $\lambda(t, x, \xi)=I+\tilde{\lambda}(t, x, \xi)$ and $\lambda^{\prime}(t, x, \xi)=I-\tilde{\lambda}(t, x, \xi)$ where $I$ is the identity, and let $\tilde{\Lambda}(t)=\tilde{\lambda}\left(t, x, D_{x}\right)$, $\Lambda(t)=\lambda\left(t, x, D_{x}\right), \Lambda^{\prime}(t)=\lambda^{\prime}\left(t, x, D_{x}\right)$ denote the corresponding pseudodifferential operators. Then we have the following

LEMmA 4.1. Under the assumptions above there exists $c_{o}(t) \in\left(S^{0}\right)^{2 \times 2}$ $\forall t \in[0, T]$ such that

$$
\Lambda(t)(L(t) v)=L^{d}(t) \Lambda(t) v+c_{o}(t) v
$$

where $L^{d}(t)=\ell^{d}\left(t, x, D_{x}\right)$ and

$$
\ell^{d}(t, x, \xi)=\left(\begin{array}{cc}
h(x, \xi)-b_{11}(t, x, \xi) & 0 \\
0 & -h(x, \xi)-b_{22}(t, x, \xi)
\end{array}\right) .
$$

PRoof. In what follows we shall denote the symbol of a pseudo-differential operator, say $Q$, by $\sigma(Q)$. Since $\Lambda^{\prime} \Lambda=I-\tilde{\Lambda}^{2}$ we have

$$
\Lambda L=\Lambda L\left(\Lambda^{\prime} \Lambda+\tilde{\Lambda}^{2}\right)=\Lambda L \Lambda^{\prime} \Lambda+\Lambda L \tilde{\Lambda}^{2} .
$$

where $\sigma\left(\Lambda L \tilde{\Lambda}^{2}\right)(t) \in\left(S^{0}\right)^{2 \times 2} \forall t \in[0, T]$. Moreover

$$
\begin{aligned}
\sigma\left(\Lambda L \Lambda^{\prime}\right)(t) & =\sigma(L-L \tilde{\Lambda}+\tilde{\Lambda} L-\tilde{\Lambda} L \tilde{\Lambda})(t) \\
& =\ell(t, ., .)+\sigma(\tilde{\Lambda} L-L \tilde{\Lambda})(t)-\sigma(\tilde{\Lambda} L \tilde{\Lambda})(t)
\end{aligned}
$$

where $\sigma(\tilde{\Lambda} L \tilde{\Lambda})(t) \in\left(S^{0}\right)^{2 \times 2} \forall t \in[0, T]$. Then we have:

$$
\sigma(\tilde{\Lambda} L-L \tilde{\Lambda})(t)=\sigma(\tilde{\Lambda} \mathscr{H}-\mathscr{H} \tilde{\Lambda})(t)+\sigma(\tilde{\Lambda} \mathscr{B}-\mathscr{B} \tilde{\Lambda})(t)
$$

where $\sigma(\tilde{\Lambda} \mathscr{B}-\mathscr{B} \tilde{\Lambda})(t) \in\left(S^{0}\right)^{2 \times 2} \forall t \in[0, T]$. Moreover, if $b$ denotes the symbol of $\mathscr{B}$ and $b^{d}$ its diagonal, we have:

$$
\sigma(\tilde{\Lambda} \mathscr{H}-\mathscr{H} \tilde{\Lambda})(t)=b(t)-b^{d}(t)+r_{0}(t),
$$

with $r_{0}(t) \in\left(S^{0}\right)^{2 \times 2}$. Denoting $r_{0}-\sigma(\tilde{\Lambda} L \tilde{\Lambda})+\sigma(\tilde{\Lambda} \mathscr{B}-\mathscr{B} \tilde{\Lambda})$ by $z$, we obtain

$$
\sigma\left(\Lambda L \Lambda^{\prime}\right)(t)=\ell(t)+b(t)-b^{d}(t)+z(t)=\ell^{d}(t)+z(t) .
$$


Denoting $Z(t) \Lambda(t)+\Lambda(t) L(t) \tilde{\Lambda}^{2}(t)$ by $C_{o}(t)$ and its symbol by $c_{0}(t)$, we prove our claim in view of (16), (17).

Now we derive energy estimates for the diagonalized system. Define

$$
k(x, \xi)=\left(\begin{array}{cc}
e^{-M p(x, \xi)} & 0 \\
0 & e^{M p(x, \xi)}
\end{array}\right)
$$

where $p(x, \xi)=\langle\xi\rangle^{-1} \sum_{j=1}^{n} \xi_{j} \operatorname{arctg} x_{j}$ and $M \geq r c_{r} / c$, with $c_{r}$ as in (14), and $c$ as in (H3). Denote the corresponding operator by $K\left(x, D_{x}\right)$. Applying $K \Lambda(t)$ to (12) we get

$$
\begin{aligned}
\frac{d}{d t}\|K \Lambda(t) w(t)\|^{2}= & 2 \operatorname{Re}\left\langle K \partial_{t}(\Lambda(t) w(t)), K \Lambda(t) w(t)\right\rangle \\
=2 & \operatorname{Re}\langle K(\varepsilon \Delta \Lambda(t)-i \Lambda(t) L(t)+\varepsilon[\Lambda(t), \Delta] \\
& \left.\left.+\left[\partial_{t}, \Lambda(t)\right]\right) w(t)+K \Lambda(t) G(u(t)), K \Lambda(t) w(t)\right\rangle
\end{aligned}
$$

which, in view of Lemma 4.1, is equal to

$$
2 \operatorname{Re}\left\langle K\left(\left(\varepsilon \Delta-i L^{d}(t)\right) \Lambda(t)+r_{\varepsilon}(t)\right) w(t)+K \Lambda(t) G(u(t)), K \Lambda(t) w(t)\right\rangle
$$

where $r_{\varepsilon}(t)=\varepsilon[\Lambda(t), \Delta]+\left[\partial_{t}, \Lambda(t)\right]-i c_{o}(t)$ with $c_{o}(t) \in\left(S^{0}\right)^{2 \times 2} \forall t \in$ $[0, T]$. Since the first term in the asymptotic expansion of $\sigma([\Lambda(t), \Delta])(x, \xi)$ is

$$
\left(\begin{array}{cc}
0 & -\sum_{j=1}^{0} \xi_{j} D_{x_{j}}\left(b_{12}(t, x, \xi) / H(x, \xi)\right) \\
\sum_{j=1}^{0} \xi_{j} D_{x_{j}}\left(b_{21}(t, x, \xi) / H(x, \xi)\right) & 0
\end{array}\right)
$$

which belongs to $\left(S^{0}\right)^{2 \times 2}$, then $c_{o}(t) \in\left(S^{0}\right)^{2 \times 2} \forall t \in[0, T]$.

Let us now examine the symbol of the diagonal matrix $K\left(\varepsilon \Delta-i L^{d}(t)\right)-$ $\left(\varepsilon \Delta-i L^{d}(t)\right) K$. A simple calculation shows that it is of the form

$$
M\left(\begin{array}{cr}
\{p, H\}(x, \xi) & \\
+2 \varepsilon i \xi . \nabla_{x} p(x, \xi) & 0 \\
+s_{o}(t, x, \xi) & \\
& \begin{array}{r}
\{p, H\}(x, \xi) \\
0
\end{array} \\
& -2 \varepsilon i \xi . \nabla_{x} p(x, \xi) \\
& +\tilde{s}_{o}(t, x, \xi)
\end{array}\right) k(x, \xi)
$$


with $s_{o}(t), \tilde{s}_{o}(t) \in S^{0}$. Thus

$$
\begin{aligned}
& \frac{d}{d t}\|K \Lambda(t) w(t)\|^{2} \\
& \leq-2 \operatorname{Re}\left\langle\left(i L^{d}(t)-\varepsilon \Delta+M\{H, p\}\right) K \Lambda(t) w(t), K \Lambda(t) w(t)\right\rangle \\
& \quad+\left(C_{\varepsilon}^{\prime}\|w(t)\|+\|K \Lambda(t) G(u)\|\right)\|K \Lambda(t) w(t)\|
\end{aligned}
$$

In view of the assumption (H3) and of (14), we have

$$
\operatorname{Im} b_{k k}(t, x, \xi)+M\{H, p\}(x, \xi) \geq\left(-c_{r} r+M c\right)\langle x\rangle^{-2}|\xi| \geq 0,
$$

for $k=1,2$. Then by applying the sharp Gårding inequality we obtain

$$
\operatorname{Re}\left\langle\left(i L^{d}(t)+M\{H, p\}\right) K \Lambda(t) w(t), K \Lambda(t) w(t)\right\rangle \geq-\tilde{C}_{r}\|K \Lambda(t) w(t)\|^{2},
$$

for some $\tilde{C}_{r}>0$. Hence

$$
\begin{aligned}
& -2 \operatorname{Re}\left\langle\left(i L^{d}(t)-\varepsilon \Delta+M\{H, p\}\right) K \Lambda(t) w(t), K \Lambda(t) w(t)\right\rangle \\
& \quad \leq 2 \tilde{C}_{r}\|K \Lambda(t) w(t)\|^{2}-2 \varepsilon\|\nabla K \Lambda(t) w(t)\|^{2} \leq 2 \tilde{C}_{r}\|K \Lambda(t) w(t)\|^{2} .
\end{aligned}
$$

Then we get

$$
\begin{aligned}
\frac{d}{d t}\|K \Lambda(t) w(t)\|^{2} \leq 2 & \tilde{C}_{r}\|K \Lambda(t) w(t)\|^{2} \\
& +\left(C_{\varepsilon}^{\prime}\|w(t)\|+\|K \Lambda(t) G(u)\|\right)\|K \Lambda(t) w(t)\|
\end{aligned}
$$

\section{End of the proof of the theorem}

Let

$$
\begin{aligned}
\tilde{E}(u(t))= & \sum_{|\alpha|=m+\ell}\left\|K \Lambda(t) \partial_{x}^{\alpha} u(t)\right\|+\sum_{j=1}^{n} \sum_{|\alpha|=m+1}\left\|K \Lambda(t)\left(x_{j} \partial_{x}^{\alpha} u(t)\right)\right\| \\
& +\sum_{|\alpha|=m}\left\|K \Lambda(t)\left(|x|^{2} \partial_{x}^{\alpha} u(t)\right)\right\|
\end{aligned}
$$

Let $\varepsilon \in] 0,1]$ and let $u_{\varepsilon} \in \mathscr{C}\left([0, T] ; \Xi^{m, \ell}\right)$ be a solution of (4) such that $\sup _{t \in[0, T]}\left\|u_{\varepsilon}(t)\right\|_{\Xi^{m-1, \ell}} \leq r$. Let

$$
E\left(u_{\varepsilon}(t)\right)=\tilde{E}\left(u_{\varepsilon}(t)\right)+\left\|u_{\varepsilon}(t)\right\|_{\Xi^{m-1, \ell}} .
$$

As in the proof of (4.3) in [3], one can see that $E\left(u_{\varepsilon}(t)\right)$ is equivalent to $\left\|u_{\varepsilon}(t)\right\|_{\Xi^{m, \ell}}$; specifically, if $\left\|u_{\varepsilon}(t)\right\|_{\Xi^{m-1, \ell}} \leq r$, then there exists $M_{r}>1$ such that

$$
M_{r}^{-1}\left\|u_{\varepsilon}(t)\right\|_{\Xi^{m, \ell}} \leq E\left(u_{\varepsilon}(t)\right) \leq M_{r}\left\|u_{\varepsilon}(t)\right\|_{\Xi^{m, \ell}} .
$$


Now from (19) and (15) we have

$$
\frac{d}{d t}\|K \Lambda(t) w(t)\|^{2} \leq C_{r}^{* *} E\left(u_{\varepsilon}(t)\right)\|K \Lambda(t) w(t)\|,
$$

and summing up on $\varphi(x)$ and $\alpha$ we obtain

$$
\frac{d}{d t} \tilde{E}\left(u_{\varepsilon}(t)\right) \leq C_{r}^{*} E\left(u_{\varepsilon}(t)\right) .
$$

Thus we finally obtain

$$
E\left(u_{\varepsilon}(t)\right) \leq E\left(u_{o}\right) e^{C_{r}^{*} t}
$$

with $C_{r}^{*}$ which is independent of $\left.\left.\varepsilon \in\right] 0,1\right]$. Then there exists a time $T>0$ such that $\left\{u_{\varepsilon}\right\}_{\varepsilon \in] 0,1]}$ is bounded in $\mathscr{C}\left([0, T] ; \Xi^{m, \ell}\right)$, and thus by a standard argument we get a solution $u(t) \in \Xi^{m, \ell} \forall t \in[0, T]$ of (3).

\section{REFERENCES}

1. Agliardi, R., Mari, D., On the Cauchy problem for some pseudo-differential equations of Schrödinger type, $M^{3} A S$, Math. Models Methods Appl. Sc. 6 (1996), 295-314.

2. Chihara, H., Local existence for the semilinear Schrödinger equations in one space dimension, J. Math. Kyoto Univ. 34-2 (1994), 353-367.

3. Chihara, H., Local existence for semilinear Schrödinger equations, Math. Japon. 42 (1995), $35-52$.

4. Chihara, H., Global existence of small solutions to semilinear Schrödinger equations, Comm. Partial Differential Equations 21 1\&2 (1996), 63-78.

5. Doi, S., On the Cauchy problem for Schrödinger type equations and the regularity of solutions, J. Math. Kyoto Univ. 34-2 (1994), 319-328.

6. Doi, S., Remarks on the Cauchy problem for Schrödinger-type equations, Comm. Partial Differential Equations 21 1\&2 (1996), 163-178.

7. Hara, S., A necessary condition for $H^{\infty}$-wellposed Cauchy problem of Schrödinger type equations with variable coefficients, J. Math. Kyoto Univ. 32 (1992), 287-305.

8. V. Ichinose, Sufficient conditions on $H^{\infty}$ well-posedness for Schrödinger type equations, Comm. Partial Differential Equations 9 (1984), 33-48.

9. Kato, T., Nonlinear Schrödinger equations, in Schrödinger Operators, (H. Holden, A. Jensen eds.), Lecture Notes in Phys. 345 (1989), 218-263.

10. Kitada, H., Kumano-Go, H., A family of Fourier integral operators and the fundamental solution for a Schrödinger equation, Osaka J. Math. 18 (1981), 291-360.

11. Kumano-Go, H., Pseudo-Differential Operators, 1981, MIT Press, Cambridge.

12. Mizohata, S., On the Cauchy Problem, 1985, Academic Press, New York.

DEPARTMENT OF MATHEMATICS,

UNIVERSITY OF FERRARA

VIA MACHIAVELLI, 35

I-44100 FERRARA

ITALY 